\title{
The Abnormal Open-Field Behavior of SART-Stressed Rats and Effects of Some Drugs on It
}

\author{
Taeko HATA, Yoshitaka NISHIMURA, Tomitaro KITA, \\ Eiji ITOH and Atsufumi KAWABATA \\ Department of Pharmacology, Faculty of Pharmacy, Kinki University, \\ Kowakae. Higashi-Osaka 577, Japan \\ Accepted September 10, 1988
}

\begin{abstract}
As part of an investigation on the behavioral characteristics of SARTstressed animals, an animal model of autonomic imbalance, the open-field behavior of SART-stressed (repeated cold-stressed) rats was studied and compared with that of rats exposed to other types of stress. In addition, the effects of several drugs on it were also studied. As compared with normal rats, SART-stressed rats exhibited increased locomotor activity, rearing and center-field penetration, together with decreased grooming and increased defecation, whereas they showed no significant changes in spontaneous movements in the daytime as measured by an Animex activity meter. These behavioral abnormalities were remarkably different from those due to $1-\mathrm{hr}$ cold, $48-\mathrm{hr}$ cold and repeated restraint stresses. These abnormal forms of open-field behavior due to SART stress were considerably inhibited by chlorpromazine, imipramine and neurotropin at doses having no corresponding influence on normal rats; and they were partially inhibited by alprazolam, diazepam and carpipramine at doses exerting considerable influence on normal rats. The above results show that SART-stressed rats exhibit open-field behavioral abnormalities that are different from those of rats exposed to other types of stress. Such abnormalities include excessive activity, which is considered to be caused by excessive emotionality.
\end{abstract}

Many researchers have reported that animals exposed to stress exhibit various forms of abnormal behavior. Among these characteristics, open-field behavioral abnormality includes increased locomotor activity in mice (1) resulting from environmentally induced psychological stress due to changes in light, sound, and other parameters or locomotor activity in rats (2) that increases under heat stress at $47^{\circ} \mathrm{C}$ and $52^{\circ} \mathrm{C}$, but decreases at $57^{\circ} \mathrm{C}$. In addition, Lee et al. (3) have reported that acute restraint stress and foot-shock stress increased locomotor activity in mice, which was considered to be caused mainly by an increase in center region activity, that is, movement inside the central area of the field. Levine et al. (4) and Anderson et al. (5) have further reported that rats loaded with 5 days of repeated foot-shock stress exhibited decreased locomotor activity and rearing cou- pled with increased defecation. Also, Roth and Katz (6) and Katz et al. (7) have reported the results of their detailed examination of the effects of acute and chronic stresses on the open-field behavior of rats.

The present authors have been investigating, from various angles, the symptomatic picture in SART (specific alternation of rhythm in temperature)-stressed animals (8), a model of autonomic imbalance of the parasympathetic-nerve priority type (9). SART-stressed animals, in addition to having various physiological abnormalities (10-15). have been reported to exhibit a decreased acetylcholine content, increased activity of related enzymes $(16,17)$, and increased contents of norepinephrine and dopamine in various brain areas (18). Since these neurotransmitters are known to be deeply associated with the manifestation of various forms of 
behavior, and such animals also exhibit abnormal electrocorticograms (15), the abnormal behavior of SART-stressed animals can be predicted with reasonable accuracy.

In this context, the present study was carried out to examine the behavioral characteristics of animais with this form of stress. We studied the open-field behavior of SARTstressed rats, and we furthermore performed comparative examinations using results obtained from rats loaded with other types of stress. In addition, the influence of several drugs on the open-field behavioral abnormality of SART-stressed rats was studied.

\section{Materials and Methods}

Male Wistar rats (Shizuoka Laboratory Animal Center), weighing between 200 and $250 \mathrm{~g}$, were used. They were usually housed in groups of five each in a wire-netting cage $(38 \times 25 \times 17 \mathrm{~cm})$ placed in a temperatureand light-controlled room $\left(24 \pm 1^{\circ} \mathrm{C}\right.$, with a 12-hr light-dark cycle starting at 07:00) with free access to food (MF, Oriental Yeast) and water at all times.

SART stress: According to the procedures reported previously (19), rats were exposed to an environmental temperature alternating between $24^{\circ} \mathrm{C}$ and $-3{ }^{\circ} \mathrm{C}$ at 1 -hr intervals from 09:00 to $16: 00$ and then kept at $-3^{\circ} \mathrm{C}$ from 16:00 to 09:00 the following day. For this purpose, 2 cages were kept at $24^{\circ} \mathrm{C}$ and $-3^{\circ} \mathrm{C}$, respectively, and only the rats were transferred at the appointed time from one cage to the other. These procedures were repeated for 5 consecutive days. Stress loading was discontinued on the 6th morning, and the animals were subjected to the experiment.

Cold stress: Rats were kept at $-3^{\circ} \mathrm{C}$ for $1 \mathrm{hr}$ or $48 \mathrm{hr}$. Experiments were started immediately after discontinuation of the stress loading.

Repeated restraint stress: Rats were denied water and food, and they were restrained using a metal net from 18:00 to 09:00 the following day. This procedure was repeated for 5 consecutive days, and the experiment was conducted on the day of final stress loading. Animals in a control group were only denied water and food according to the same schedule.

Open-field test: The open-field test ap- paratus used was a cylindrical container, having a diameter of $60 \mathrm{~cm}$ and a height of $42 \mathrm{~cm}$, with internal walls painted black (BRS/LVE PAC-001. Tech SerV). The floor of this apparatus was a stainless steel net, which was removable. Six photobeams were placed $4 \mathrm{~cm}$ above the floor; these divided the floor area into 16 sections. Two 40-W fluorescent lamps were fitted $250 \mathrm{~cm}$ above the floor, and the illuminance at the floor level was 120 !ux. The background noise due to the room air conditioner was 65 to $70 \mathrm{~dB}$. Measurements were done between 12:00 and 17:00 daily. Upon completion of measurement with every individual rat, the floor was removed, washed with water and thoroughly dried, and then put back for use with the next animal.

The open-field test adopted was based on the procedure described by Roth and Katz (6) and Katz et al. (7). Briefly, a rat was placed in the corner of the field with its head toward the wall, and measurements were conducted for $15 \mathrm{~min}$ at 3 -min intervals. The measurements included 5 parameters: locomotor activity (the frequency with which the animal crossed the photobeams), rearing activity (the frequency with which the animal stood up on its hind legs), time spent in grooming (time in seconds required for the animal to groom), center-field penetration (the frequency with which the animal entered a circle with a radius of $15 \mathrm{~cm}$ drawn in the center of the field), and defecation score (the total number of pellets excreted in the 15 -min period).

Measurement of the amount of spontaneous motor activity: Using an Animex activity meter (Type S, Farad), the amount of spontaneous motor activity of individual animals was automatically measured. Rats had been placed previously in a plastic cage $(38 \times$ $22 \times 15 \mathrm{~cm}$ ) for measuring the amount of motor activity and acclimatized for $60 \mathrm{~min}$. after which the measurement was performed for $120 \mathrm{~min}$. All measurements were done in a soundproof chamber starting at 12:00. The illuminance in the cage was $100 \mathrm{lux}$, and the background noise due to the room air conditioner was 55 to $60 \mathrm{~dB}$.

Drugs: Anxiolytics, alprazolam (Takeda) and diazepam (Wako); antipsychotics, chlorpromazine hydrochloride (Shionogi) and 
carpipramine dihydrochloride monohydrate (Yoshitomi); an antidepressant, imipramine hydrochloride (Sigma); and a sedative analgesic, neurotropin (an extract from the inflamed skin of vaccinia virus-inoculated rabbits, Nippon Zoki, $10 \mathrm{mg} / \mathrm{ml}$ ), were used. Alprazolam and diazepam were suspended in $0.5 \%$ CMC- $\mathrm{Na}$ solution and administered orally to rats. Chlorpromazine, carpipramine and imipramine were dissolved in $0.9 \%$ physiological saline, and administered i.p. to rats. In the case of neurotropin, the undiluted solution was given i.p. to rats. Control animals received the vehicle only. All drugs were administered once $60 \mathrm{~min}$ before the initiation of the open-field test, when steady effects were obtained in preliminary experiments.

It should be mentioned here that neurotropin is a sedative analgesic, which is clinically effective for treating patients with chronic pain; and in animal experiments, it has been reported to effectively moderate most of the abnormal symptoms observed in SARTstressed animals including abnormalities in ECG (12) and EEG (20), alterations in the contents of acetylcholine (17) and catecholamines (21), abnormal blood flow $(11,14)$. hyperalgesia (22), and so on.

Statistical analysis: Data obtained were expressed in terms of the mean \pm S.E. The significance of differences was assessed by Student's $t$-test or Duncan's multiple-range test.

\section{Results}

1. Open-field behavior and spontaneous motor activity in SART-stressed rats: Figure 1 illustrates the results obtained for every 3min interval in the open-field test carried out on non-stressed and SART-stressed rats. As can be seen. SART-stressed rats exhibited significant increases in locomotor activity and significant decreases in the time spent in grooming during the first $12 \mathrm{~min}$. Rearing activity increased only during the first $6 \mathrm{~min}$, and center-field penetration showed a significant increase from 9 to $12 \mathrm{~min}$ after the start of measurement.

Total amounts for these 4 parameters and defecation scores for $15 \mathrm{~min}$ are shown in Table 1. SART-stressed rats exhibited significant changes in all 5 parameters.

The amount of spontaneous motor activity for $2 \mathrm{hr}$ in the daytime was $704.9 \pm 68.3$ counts $(n=12)$ in non-stressed rats and $798.7 \pm 39.0$ counts $(n=10)$ in SART-stressed rats. These motor activities showed practically no significant difference between the non-stressed and the SART-stressed rats.

2. Open-field behavior in cold- and re-
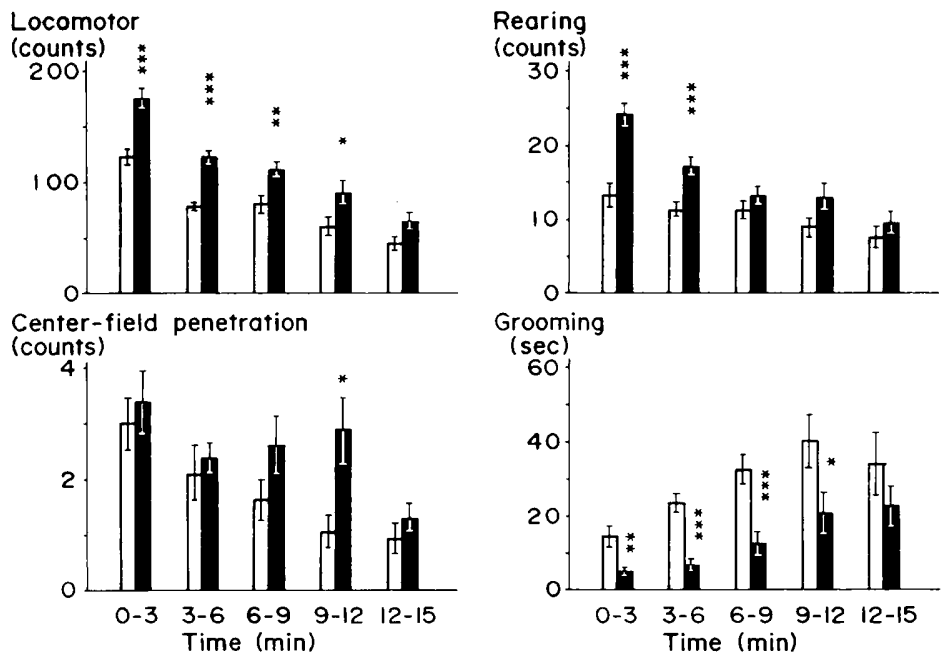

Fig. 1. Open-field behavior in non-stressed and SART-stressed rats. $\square$, Non-stress: SART stress. All parameters are shown as total amounts for each 3 -min interval. Data show the mean $\pm S$.E. from 16 rats. ${ }^{*} \mathrm{P}<0.05,{ }^{* *} \mathrm{P}<0.01,{ }^{* *} \mathrm{P}<0.001$ ( $t$-test). 
Table 1. Changes in open-field behavior in SART-stressed rats

\begin{tabular}{lcc}
\hline \multicolumn{1}{c}{ Parameters } & Non-stressed rats & SART-stressed rats \\
\hline Locomotor activity (counts) & $388.4 \pm 16.0$ & $566.1 \pm 25.0^{* * *}$ \\
Rearing activity (counts) & $52.1 \pm 4.6$ & $76.9 \pm 4.3^{* * *}$ \\
Center-field penetration (counts) & $8.8 \pm 1.2$ & $12.6 \pm 1.3^{*}$ \\
Time spent in grooming (sec) & $145.2 \pm 11.0$ & $68.2 \pm 8.5^{* * *}$ \\
Defecation score (pellets) & $3.0 \pm 0.6$ & $5.3 \pm 0.3^{* *}$ \\
\hline
\end{tabular}

Data show the total amounts for $15 \mathrm{~min}$ as the mean \pm S.E. from 16 rats. $\quad{ }^{*} P<0.05,{ }^{* *} P<0.01,{ }^{* * *} P<0.001$ ( $t$-test).
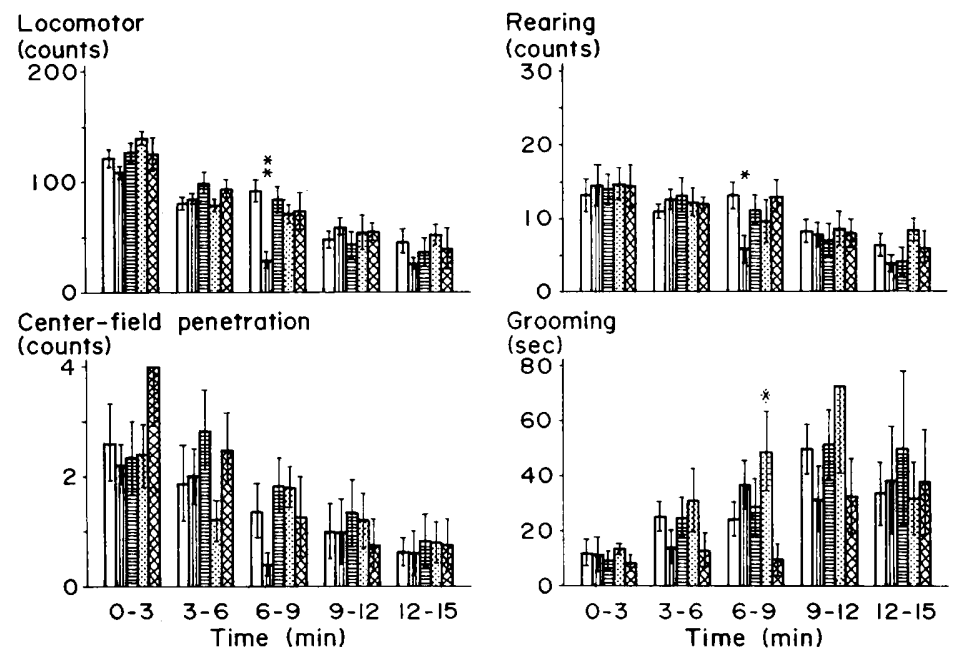

Fig. 2. Open-field behavior in rats exposed to cold stress or repeated restraint stress. $\square$. Non-stress:

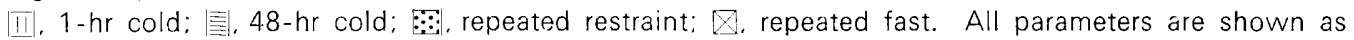
total amounts for each 3 -min interval. Data show the mean \pm S.E. from $4-8$ rats. ${ }^{*} P<0.05,{ }^{* *} P<0.01$. compared to the non-stress group. $※ p<0.05$, compared to the repeated fast group (Duncan's test).

peated restraint-stressed rats: Figure 2 shows the results obtained from the open-field test conducted on rats exposed to other types of stress. The 1-hr cold stress group exhibited decreases in locomotor and rearing activities for a 3-min period only during 6-9 $\mathrm{min}$, and no significant change in other parameters in comparison with the controls was apparent. The 48-hr cold stress, repeated restraint, and fast groups showed practically no difference from the non-stressed control group. The repeated restraint group exhibited an increase in time spent in grooming compared with the water and food-denied control group. None of these stressed groups showed any significant change in the total amounts of these 4 parameters in a 15 -min period. As to the defecation score, neither 1 -hr nor 48-hr cold stress $(2.4 \pm 1.0$ and $1.7 \pm 0.9$ pellets $/ 15 \mathrm{~min}$, respectively) produced any change. However, the restraint stress group exhibited a significantly $(P<0.01)$ increased defecation score, $6.8 \pm 0.9$ pellets $/ 15 \mathrm{~min}$, compared with that in non-stressed rats, $2.1 \pm 0.8$, while the corresponding control group, which had been denied water and food, likewise exhibited an increase in the score, resulting in approximately the same value, i.e., $7.1 \pm 1.4$.

3. Effects of drugs on the open-field behavioral abnormality in SART-stressed rats: The effects of drugs were evaluated on the basis of locomotor and rearing activities during the initial $3 \mathrm{~min}$ and $15 \mathrm{~min}$, and from center-field penetration, grooming and defecation score for a 15-min period.

Table 2 shows the action of the anxiolytics, 


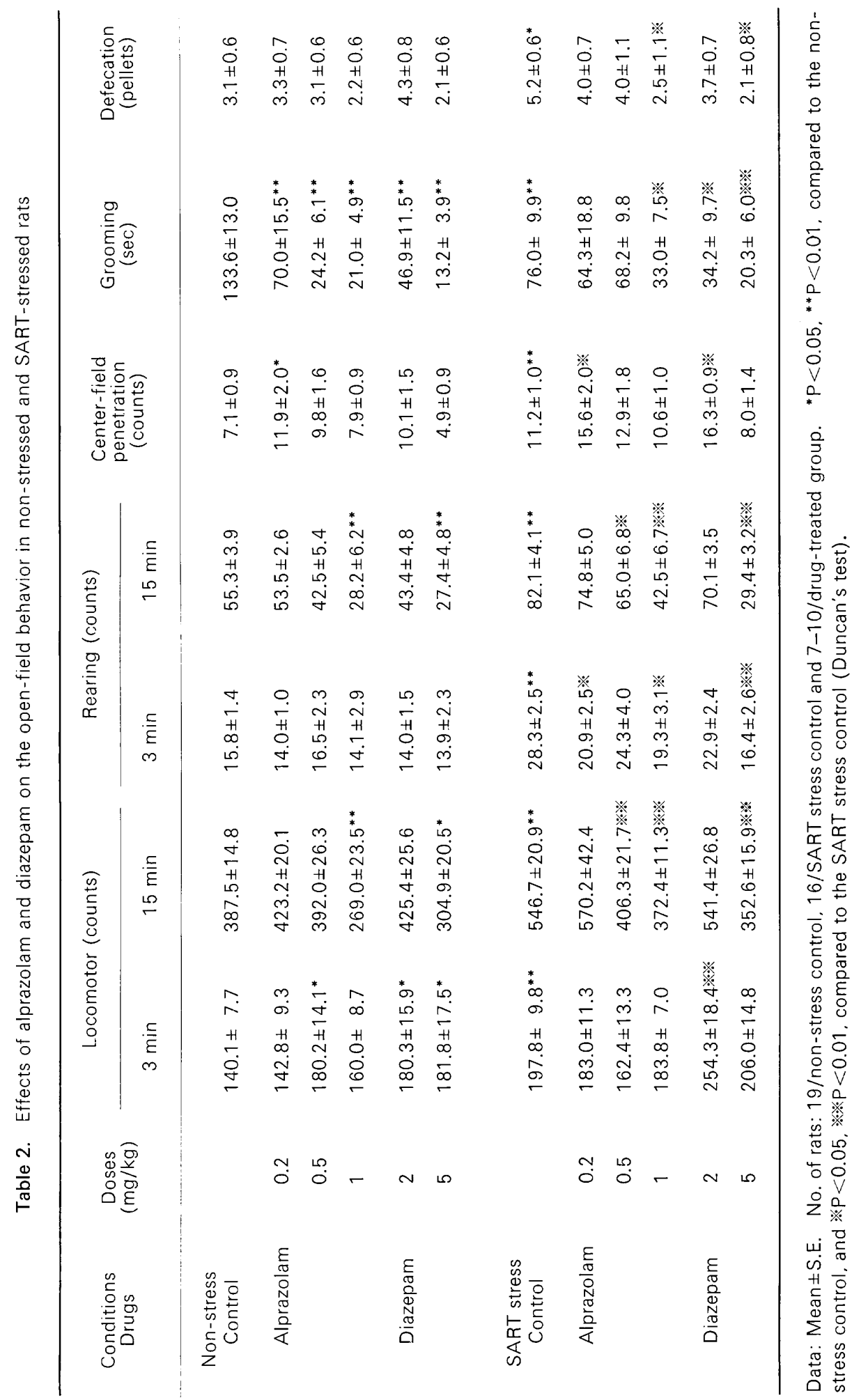




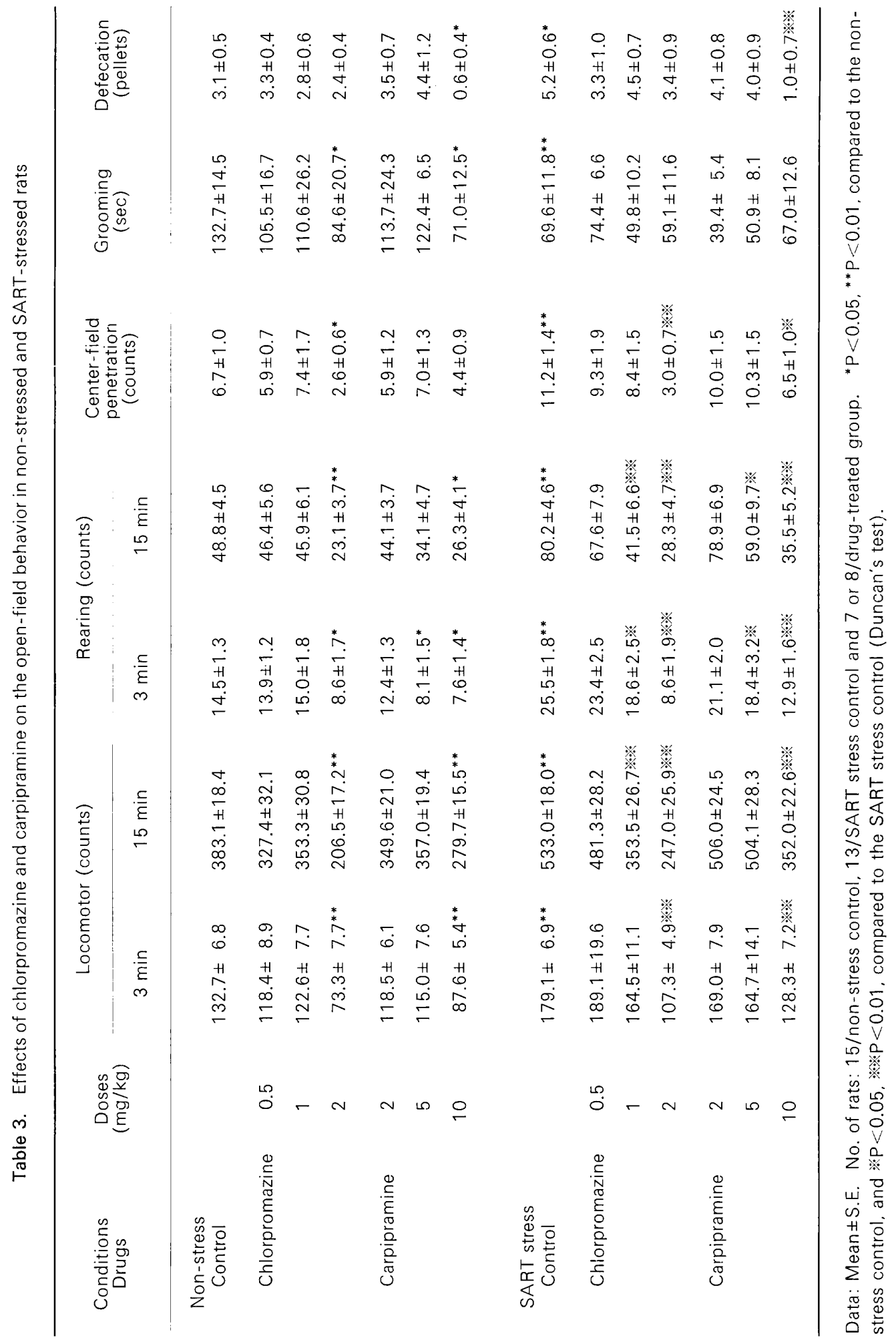




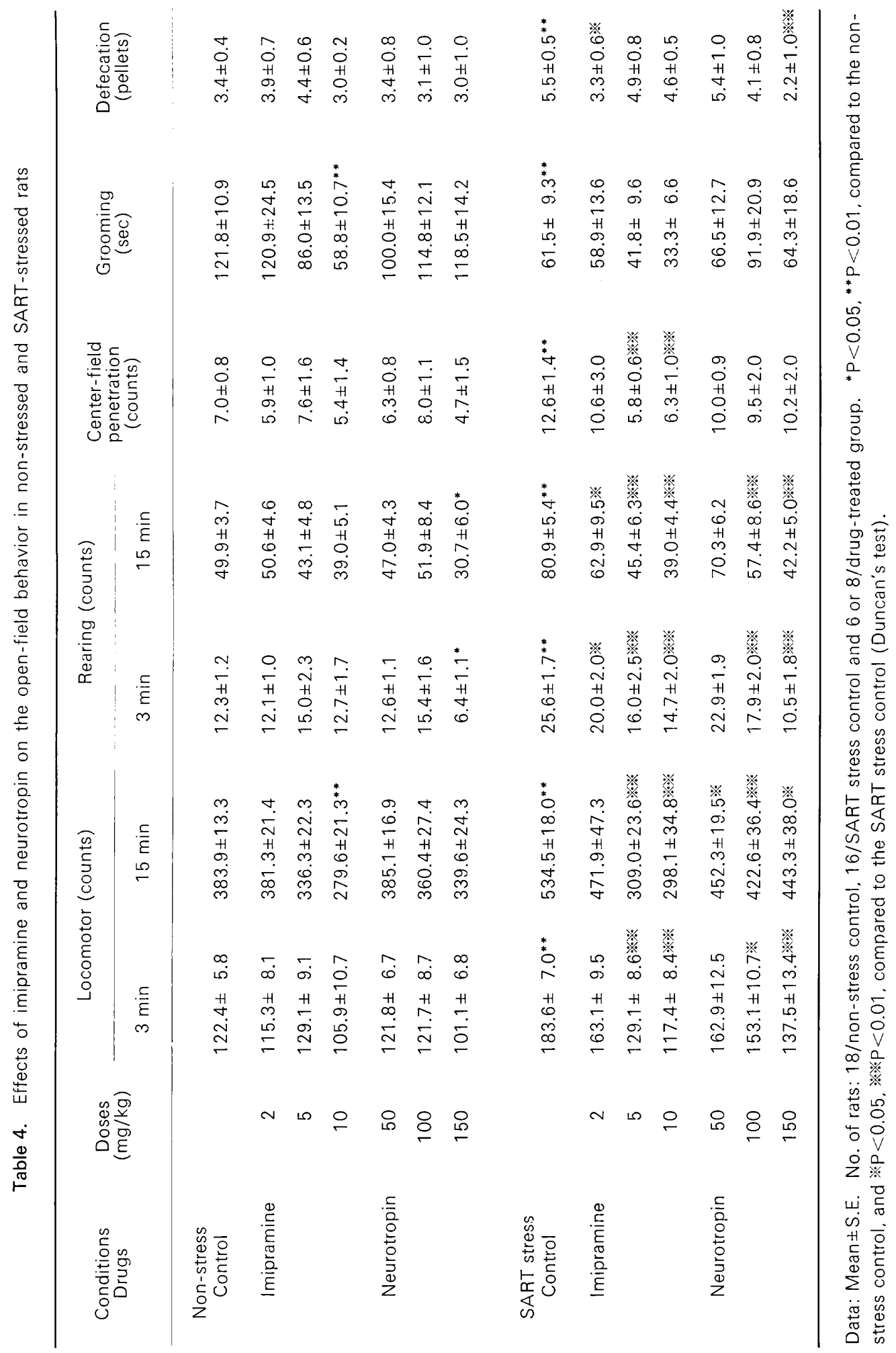


aiprazolam and diazepam. In bothnon-stressed rats and SART-stressed rats, alprazolam caused dose-dependent decreases in locomotor, rearing and grooming activities for 15 min in addition to an increase in center-field penetration at a lower dose. Also, in SARTstressed rats, a!prazolam produced a dosedependent reduction in both the 3 -min rearing activity and defecation score previously increased by the stress. Diazepam showed a similar action to alprazolam on open-field behavior in non-stressed and SART-stressed rats.

Thus, both alprazolam and diazepam counteracted the increases in 3-min rearing and defecation due to SART stress, but affected other open-field behavioral parameters in SART-stressed rats in a similar fashion to those in unstressed rats.

Table 3 shows the action of the antipsychotics, chlorpromazine and carpipramine. Chlorpromazine at $0.5 \mathrm{mg} / \mathrm{kg}$ failed to affect the open-field behavior of both non-stressed and SART-stressed rats, whereas at $1 \mathrm{mg} / \mathrm{kg}$. it completely reversed the SART stressaugmented 15 -min locomotor and 3 -min and 15 -min rearing activities without influencing the behavior of non-stressed rats. When rats received a larger dose of chlorpromazine, 2 $\mathrm{mg} / \mathrm{kg}$, all parameters with the exception of defecation score were decreased in both the unstressed and stressed groups. On the other hand, carpipramine suppressed most of the open-field behavioral parameters in a similar fashion in both non-stressed and stressed rats.

Thus, $1 \mathrm{mg} / \mathrm{kg}$ chlorpromazine blocked the SART stress-induced increases in locomotor and rearing activities without producing any notable influence on the behavior of unstressed rats.

Finally, the effects of the antidepressant imipramine and the sedative ana!gesic neurotropin are presented in Table 4. Imipramine, $10 \mathrm{mg} / \mathrm{kg}$, produced only decreases in 15 min locomotor activity and grooming in unstressed rats. In SART-stressed rats, on the other hand, imipramine decreased all parameters, except for grooming, previous!y increased by the stress.

Neurotropin at doses of 50 and $100 \mathrm{mg} / \mathrm{kg}$ produced hardly any effect on the openfield behavior of non-stressed rats, but caused dose-dependent decreases in locomotor and rearing activities in SART-stressed rats. In addition, neurotropin at $150 \mathrm{mg} / \mathrm{kg}$ reduced only rearing activity but did not affect any other parameters in non-stressed rats. However, it suppressed locomotor and rearing activities and defecation scores previously increased by SART stress.

\section{Discussion}

The open-field test (23-27) is a method which is aimed at examining the emotion of an individual animal on the basis of its behavior when it is placed in a novel environment, and it is utilized in the study of emotion or screening of psychopharmaceuticals. In this test, locomotor and rearing activities are accepted as indicators for exploratory movements, while grooming is a measure of emotional stability, and defecation is regarded as an indicator of emotionality. In other words, rats with elevated emotionality exhibit increased locomotor and rearing activities, while their grooming decreases and defecation increases. On the other hand, it is known that rats whose emotionality has been extremely elevated exhibit freezing and decreased exploratory movements.

In the present study, the SART-stressed rats showed increased locomotor and rearing activities in the open-field test in comparison with non-stressed rats; i.e., they showed excessive behavioral activity. while spontaneous motor activity for a 2-hr period measured by the Animex activity meter showed no significant increase. If it is taken into consideration that this excess in activity was also seen in the early stage of observation and was accompanied by a decrease in grooming and an increase in defecation and that spontaneous motor activity was measured after $1-\mathrm{hr}$ acclimatization to the apparatus, this can be considered to have been due to elevation of emotionality. SART-stressed rats showed nearly equal amounts of center-field penetration for all 3 -min periods until $12 \mathrm{~min}$ after the start of measurement, while the center-field penetration of unstressed rats decreased gradually with time. In addition, time spent in grooming by SART-stressed rats was limited throughout the measurement period. especially up to $12 \mathrm{~min}$, compared with that 
by non-stressed rats. Ambulation in the center or inner area of the apparatus is thought to be related to timidity $(25,28)$. However, the increased center-field penetration of SARTstressed rats may be due to higher activity and/or emotionality; and such rats are postulated to be resistant to acclimatization to a new environment.

The above-observed open-field behavioral abnormalities in SART-stressed rats were distinctly different from those observed in 1 - hr or 48-hr cold stress or from those observed in repeated restraint stress studies. In the $1-\mathrm{hr}$ cold stress experiment, decreases only in locomotor and rearing activities were observed, whereas no abnormality was seen in any of the test parameters in the 48-hr stress experiment. In other words, SART stress, which results from exposure to a form of pulse-like alternation between standard and cold environments, is greatly different from exposure to stress in the form of a simple cold environment. Furthermore, from the fact that while repeated restraint stress increased defecation, the feeding time-restricted control group also showed increased defecation, resulting in similar values, it is suggested that the increased defecation was not the result of restraint stress but rather the night-time restriction of food intake. Grooming activity of restraint-stressed rats increased, in contrast to the decrease seen in SART-stressed rats.

Roth and Katz (6) and Katz et al. (7) observed in their open-field test a transient increase in locomotor activity, shortening of initial movement latency, increase in centerfield penetration, increase in grooming, and decrease in defecation under acute stress consisting of combined sound and light. However, they did not find any significant difference in rearing. On the other hand, rats loaded for 21 days with stress that was altered every day (shock, fasting, tail-pinching, or cold-water swimming) exhibited decreased locomotor activity, rearing, and center-field penetration, coupled with an increase in defecation score.

When these reports are considered in combination with those mentioned in the introduction and also our present results observed in rats loaded with some other types of stress, the abnormal forms of open-field behavior seen in SART-stressed rats seem to be unique and considerably different from those observed in rats given other types of stress. Also, these abnormalities are those observed during the period of time when no change occurred in the amount of spontaneous motor activity. Therefore, it can be postulated that the abnormal behavior of SART-stressed rats observed in the openfield test is manifested when stimulation accompanied by anxiety or fear is given: i.e., when the animals are placed into an open field. which is a novel environment. Such a hypothesis is supported by the previous findings that SART-stressed animals exhibited hypersensitivity to external stimuli in the galvanic skin response test (8) and resting-arousal electrocorticograms with lowvoltage fast waves (15). In future studies, it seems necessary to investigate motor activity during the night, which is intrinsic to rat behavior, and the circadian rhythm.

We subsequently focused our attention on the effect of drugs on the open-field behavior of unstressed and SART-stressed rats.

The observation that alprazolam and diazepam produced a decrease in locomotor activity following an increase in the initial 3 -min period in non-stressed rats is in agreement with previous reports concerning benzodiazepines in the open-field test described by other researchers (29-32). Also, chlorpromazine and carpipramine, which have been reported to reduce exploratory behavior in rats $(30,31,33)$ and locomotor activity in mice (34), produced marked suppression of behavioral activities at relatively higher doses in the present study. Imipramine at the highest dose decreased locomotion, but did not affect rearing in non-stressed rats, supporting the evidence reported by Kulkarni and Dandiya (35). Thus, the results obtained in unstressed rats are compatible with the findings reported previously.

In SART-stressed rats, chlorpromazine and imipramine suppressed the excessive behavioral activity due to the stress at dose levels that had no influence on the behavior of unstressed rats. It is well-established that the former drug blocks dopamine receptors (36) and that the latter inhibits the reuptake of monoamines, resulting in enhanced func- 
tion of monoaminergic neurons (37) in contrast to the former drug. In addition, SARTstressed rats have been reported to exhibit increased levels of norepinephrine and dopamine in various brain areas (18). Therefore, it would appear difficult for the behavioral abnormality due to this form of stress to be elucidated only by association with alterations in the function of the catecholaminergic system. Resolution of the mechanism responsible for the SART stress-induced behavioral abnormality will require more detailed studies concerning the possible involvement of other neurotransmitters including 5-hydroxytryptamine, acetylcholine, and so on.

Carpipramine reduced the locomotor and rearing activities increased by stress only at dose levels which produced decreases in those activities in unstressed animals. It has been said that carpipramine has a similar action to chlorpromazine, but that its distribution in the brain is slight after administration, unlike chlorpromazine (38). Such a profile might have at least been partly the reason why its mode of action appeared to be different from that of chlorpromazine in this study.

Alprazolam and diazepam were effective in reducing the behavioral abnormality in SARTstressed rats only at dose levels which produced some influence on the behavior of unstressed rats. Nevertheless, the fact that these drugs inhibited the increased defecation due to SART stress appears to refiect the presence of elevated emotionality in these stressed rats.

Neurotropin has been reported to be effective for moderating most of the abnormal SART stress-induced symptoms $(11,12,14$. 17, 20). Similarly, the present study showed that neurotropin blocked the increased locomotor and rearing activities and defecation caused by SART stress without influencing the behavior of non-stressed rats. Although the mechanism involved still remains to be elucidated, it appears to be at least different from that of anxiolytics such as alprazolam and diazepam.

\section{References}

1 Freeman, G.B. and Thurmond, J.B.: Brain amines and effects of chlordiazepoxide on motor activity in response to stress. Pharmacol. Biochem. Behav. 22, 665-670 (1985)

2 Galina, Z.H., Sutherland, C.J. and Amit, Z.: Effects of heat-stress on behavior and the pituitary adrenal axis in the rat. Pharmacol. Biochem. Behav. 19, 251-256 (1983)

3 Lee, E.H.Y., Tsai, M.J. and Chai, C.Y.: Stress selectively influences center region activity of mice in an open field. Physiol. Behav. 37, 659662 (1986)

4 Levine, S., Madden, J., IV, Conner, R.L., Moskal, J.R. and Anderson, D.C.: Physiological and hehavioral effects of prior aversive stimulation (preshock) in the rat. Physiol. Behav. 10, 467471 (1973)

5 Anderson, D.C., Crowell, C., Koehn, D. and Lupo, J.V.: Different intensities of unsignalled inescapable shock treatments as determinants of non-shock-motivated open field behavior: a resolution of disparate results. Physiol. Behav. 17, 391-394 (1976)

6 Roth, K.A. and Katz, R.J.: Stress, behavioral arousal, and open field activity-a reexamination of emotionality in the rat. Neurosci. Biobehav. Rev. 3, 247-263 (1979)

7 Katz, R.J., Roth, K.A. and Carroll, B.J.: A.cute and chronic stress effects on open field activity in the rat: implications for a model of depression. Neurosci. Biobehav. Rev. 5, 247-251 (1981)

8 Kita, T., Hata, T., Yoneda, R. and Okage, T.: Stress state caused by alterination of rhythm in environmental temperature, and the functional disorders in mice and rats. Folia Pharmacol. Japon. 71, 195-210 (1975) (Abs. in English)

9 Kita, T., Hata, T., Itoh, E. and Namimatsu, A.: Testing methods for vegetative syndrome in the rat and effects of Neurotropin and other drugs. Japan. J. Psychosom. Med. 23, 61-68 (1983)

10 Kita, T., Hata, T., lida, J., Yoneda, R. and Ishida, S.: Decrease in pain threshold in SART stressed mice. Japan. J. Pharmacol. 29, 479-482 (1979)

11 Hata, T., Kita, T., Namimatsu, A., Itoh, E. and Oda, Y.: Changes of blood pressure and regional blood flow in SART rats and drug actions on these changes. Folia Pharmacol. Japon. 79, 335-342 (1982) (Abs. in English)

12 Hata, T., Kita, T., Itoh, E. and Namimatsu, A.: Changes of the function in the heart of SART stressed (repeated cold stressed) mice and the action of Neurotropin on these changes. Folia Pharmaco!. Japon. 79, 482-492 (1982) (Abs. in English)

13 Kita, T., Hata, T., Itoh, E. and Harada, N.: Methacholine- and adrenaline-induced arrhythmias in repeatedly cold-stressed mice. Japan. 
J. Pharmacol. 35, 327-329 (1984)

14 Hata, T., Kita, T., Kawabata, A., Itoh, E. and Nishimura, Y.: Changes of tissue blood flow in mice loaded with SART (repeated cold) stress or restraint and water immersion stress and the effect of administered Neurotropin. Japan. J. Pharmacol. 41, 69-79 (1986)

15 Hata, T., Nishimura, Y., Kita, T., Kawabata, A. and Itoh, E.: Electrocorticogram in rats loaded with SART stress (repeated cold stress). Japan. J. Pharmacol. 45, 365-372 (1987)

16 Kita, T., Hata, T., Higashiguchi, T., Itoh, E. and Kawabata, A.: Changes of total acetylcholine content and the activity of related enzymes in SART (repeated cold)-stressed rat brain and duodenum. Japan. J. Pharmacol. 40, 174-177 (1986)

17 Hata, T., Kita, T., Higashiguchi, T. and tchida, S.: Total acetylcholine content, and activities of choline acetyltransferase and acetylcholinesterase in brain and duodenum of SART-stressed (repeated cold-stressed) rat. Japan. J. Pharmacol. 41, 475-485 (1986)

18 Hata, T., Kita, T., Kamanaka, Y., Honda, S., Kakehi, K., Kawabata, A. and Itoh, E.: Catecholamine levels in the brain of SART (repeated cold)-stressed rats. J. Auton. Pharmacol. 7, 257266 (1987)

19 Hata, T., Kita, T., Itoh, E. and Harada, N.: Experimental studies on optimal conditions of loading SART stress (repeated cold stress) upon ánimals. Japan. J. Psychosom. Med. 24, 257266 (1984)

20 Hata, T., Kita, T. and Nishimura, Y.: Effects of Neurotropin and other drugs on EEG alterations in SART-stressed (repeated cold-stressed) rats. Folia Pharmacol. Japon. 91, 163-171 (1988) (Abs. in English)

21 Hata, T., Kamanaka, Y., Kita, T., Honda, S., Itoh, E. and Kawabata, A.: Effects of Neurotropin and other drugs on changes in brain and plasma catecholamine content in SART-stressed rats. Japan. J. Pharmacol. 43, Supp. 153P (1987)

22 Kita, T., Hata, T. and Yoneda, R.: Analgesic effect of Neurotropin in mice, and comparison between analgesic effects of some drugs in SART-stressed mice and normal mice. Folia Pharmacol. Japon. 72, 573-584 (1976) (Abs. in English)

23 Denenberg, V.H.: Open-field behavior in the rat: what does it mean? Ann. N.Y. Acad. Sci. 159, 852-859 (1969)

24 Archer, J.: Tests for emotionality in rats and mice: a review. Anim. Behav. 21, 205-235 (1973)

25 Wa!sh, R.N. and Cummins, R.A.: The open-field test: a critical review. Psychol. Bull. 83, 482E04 (1976)

26 Royce, J.R.: On the construct validity of openfield measures. Psychol. Bull. 84, 1098-1106 (1977)

27 Ogawa, N.: Methods for behavioral pharmacology. Metabolism and Disease 17, 921a27 (1980) (in Japanese)

28 Imamura, G.: Emotional behavior. In Methodology for Psychological Research 5. Animal Experiment I, Edited by Yagi, B., p. 85-99, University of Tokyo Press. Tokyo (1977) (in Japanese)

29 Christmas, A.J. and Maxwell, D.R.: A comparison of the effects of some benzodiazepines and other drugs on aggressive and exploratory behaviour in mice and rats. Neuropharmacology 9, 17-29 (1970)

30 Nakama, M., Ochiai, T. and Kowa, Y.: Effects of psychotropic drugs on emotional behavior: Exploratory behavior of naive rats in holed open field. Japan. J. Pharmacol. 22, 767-775 (1972)

31 Honma, T. and Kitagawa, S.: Effects of minor tranquilizers and neuroleptics on open-field behavior in rats. Folia Pharmacol. Japon. 73, 337-345 (1977) (Abs. in English)

32 Ueki, S., Watanabe, S., Yamamoto, T., Kataoka, Y., Tazoe, N., Shibata, S., Shibata, K., Ohta, H., Kawahara, K., Takano, M., Suwandi; D., Lee, S.C. and Liou, S.Y.: Behavioral and electroencephalographic effects of alprazolam and its metabolites. Folia Pharmacol. Japon. 77, 483509 (1981) (Abs. in English)

33 Marriott, A.S. and Spencer, P.S.J.: Effects of centrally acting drugs on exploratory behaviour in rats. Br. J. Pharmacol. 25, 432-441 (1965)

34 Nakanishi, M., Tsumagari, T., Okada, T. and Kase, Y.: Pharmacological studies on 5-[3-(4piperidino-4-carbamoylpiperidino)-propyl]-10, 11-dihydro-5(H)-dibenz-(b,f)-azepine (Carpipramine) dihydrochloride, a new psychotropic agent. Arzneimittelforschung 18, 1435-1441 (1968)

35 Kulkarni, S.K. and Dandiya, P.C.: A preliminary report on the action of imipramine and antiparkinsonian agents on the open field behaviour of rats. Japan. J. Pharmacol. 24, 809-810 (1974)

36 Green, A.R. and Costain, D.W.: Pharmacology and Biochemistry of Psychiatric Disorders, Translated by Takahashi, S. and Takahashi, K... p. 134-151. Nishimura, Niigata (1984) (in Japanese)

37 Green, A.R. and Costain, D.W.: Pharmacology and Biochemistry of Psychiatric Disorders, 
Translated by Takahashi, S. and Takahashi, K., p. 82-102, Nishimura, Niigata (1984) (in Japanese)

38 Nakanishi, M., Kato, Y., Setoguchi, M., Tsuda, A. and Yasuda, H.: Studies on psychotropic drugs. III. Metabolic fate and effects on serotonin levels in brain of carpipramine. Yakugaku Zasshi 90, 197-203 (1970) (Abs. in English) 